PROCEEDINGS OF THE

AMERICAN MATHEMATICAL SOCIETY

Volume 140, Number 9, September 2012, Pages 3025-3033

S 0002-9939(2012)11231-6

Article electronically published on January 23, 2012

\title{
A CONSTRUCTION OF POLYNOMIALS WITH SQUAREFREE DISCRIMINANTS
}

\author{
KIRAN S. KEDLAYA
}

(Communicated by Matthew A. Papanikolas)

\begin{abstract}
For any integer $n \geq 2$ and any nonnegative integers $r, s$ with $r+2 s=n$, we give an unconditional construction of infinitely many monic irreducible polynomials of degree $n$ with integer coefficients having squarefree discriminant and exactly $r$ real roots. These give rise to number fields of degree $n$, signature $(r, s)$, Galois group $S_{n}$, and squarefree discriminant; we may also force the discriminant to be coprime to any given integer. The number of fields produced with discriminant in the range $[-N, N]$ is at least $c N^{1 /(n-1)}$. A corollary is that for each $n \geq 3$, infinitely many quadratic number fields admit everywhere unramified degree $n$ extensions whose normal closures have Galois group $A_{n}$. This generalizes results of Yamamura, who treats the case $n=5$, and Uchida and Yamamoto, who allow general $n$ but do not control the real place.
\end{abstract}

\section{INTRODUCTION AND RESULTS}

Throughout this paper, fix an integer $n \geq 2$, and let $P(x)$ denote a monic polynomial of degree $n$ with integer coefficients. The discriminant $\Delta(P)$ is the integer with the property that if we factor $P(x)=\left(x-r_{1}\right) \cdots\left(x-r_{n}\right)$ over some algebraically closed field, then

$$
\Delta(P)=\prod_{1 \leq i<j \leq n}\left(r_{i}-r_{j}\right)^{2} .
$$

Since $\Delta(P)$ is symmetric in the roots of $P$, it can be expressed as a polynomial in the coefficients of $P$; this polynomial has integer coefficients and turns out to be irreducible.

When $P$ is irreducible and the integer $\Delta(P)$ is squarefree, the number field $K=\mathbb{Q}[x] /(P(x))$ has ring of integers $\mathbb{Z}[x] /(P(x))$, the Galois closure $L$ of $K$ has Galois group $S_{n}$, and $L$ is everywhere unramified over its subfield $\mathbb{Q}(\sqrt{\Delta(P)})$ (see [6], or [12, Theorem 1] for a slightly stronger statement). These properties suggest the question of how often $\Delta(P)$ is squarefree. When the coefficients of $P$ are chosen suitably randomly, this is expected to occur with probability $\prod_{p} a_{p}$ where $p$ runs over prime numbers and $a_{p}$ denotes the probability that $\Delta(P)$ is not divisible by

Received by the editors March 29, 2011.

2010 Mathematics Subject Classification. Primary 11C08; Secondary 11R29.

The author was supported by NSF CAREER grant DMS-0545904, DARPA grant HR001109-1-0048, MIT (NEC Fund, Green Career Development Professorship), and UC San Diego (Warschawski Professorship). 
$p^{2}$. These probabilities have been computed by Brakenhoff [1]:

$$
a_{p}= \begin{cases}\frac{1}{2}, & p=2, n \geq 2 \\ 1-\frac{1}{p^{2}}, & p>2, n=2 \\ 1-\frac{2}{p^{2}}+\frac{1}{p^{3}}, & p>2, n=3 \\ 1-\frac{1}{p}+\frac{(p-1)^{2}\left(1-(-p)^{-n+2}\right)}{p^{2}(p+1)}, & p>2, n \geq 4 .\end{cases}
$$

Unfortunately, while it is easy to prove by sieving arguments that a randomly chosen integer is squarefree with the expected probability of $6 / \pi^{2}$ (see for instance [9, 18.6]), it seems quite difficult to prove that a polynomial takes squarefree values with the expected probability unless the degree is very small compared to the number of variables. For example, for univariate polynomials, this is known in all degrees under the $a b c$ conjecture by a theorem of Granville [7, but unconditionally only up to degree 3 by work of Hooley [11. Granville's conditional theorem was extended to multivariate polynomials by Poonen [15]; that result implies that under the $a b c$ conjecture, $\Delta(P)$ is squarefree with the expected probability. This remains true, with a suitably adjusted probability, if one imposes local conditions at finitely many places (including the infinite place).

However, without assuming any conjectures, it is not trivial to establish even the existence of infinitely many polynomials of a given degree with squarefree discriminant. This is given by the following theorem.

Theorem 1.1. Let $n \geq 2$ be an integer, let $r, s$ be nonnegative integers with $r+2 s=$ $n$, and let $S$ be a finite set of primes. Then there exist infinitely many monic irreducible polynomials $P(x)$ of degree $n$ with integer coefficients having exactly $r$ real roots, such that $\Delta(P)$ is squarefree and not divisible by any of the primes in $S$. More precisely, for some $c>0$ (depending on $n$ and $S$ ), the number of distinct squarefree discriminants produced in the range $[-N, N]$ is at least $c N^{1 /(n-1)}$.

The key idea in the proof is to construct $P$ in a special way to make squarefree sieving on the discriminant easier. For $a_{1}, \ldots, a_{n-1}, b \in \mathbb{Q}$, put

$$
\begin{aligned}
Q_{a}(x) & =n\left(x-a_{1} / n\right)\left(x-a_{2}\right) \cdots\left(x-a_{n-1}\right), \\
P_{a, b}(x) & =b+\int_{0}^{x} Q_{a}(t) d t, \\
\Delta_{a, b} & =\Delta\left(P_{a, b}\right) .
\end{aligned}
$$

Then $\Delta_{a, b}$ is (up to sign) the product of the evaluations of $Q_{a}$ at the roots of $P_{a, b}$, which is (up to sign) the resultant of $P_{a, b}$ and $Q_{a}$. However, one may also compute the resultant by evaluating $P_{a, b}$ at each root of $Q_{a}$ :

$$
\Delta_{a, b}= \pm\left(n^{n} P_{a, 0}\left(a_{1} / n\right)+n^{n} b\right) \prod_{i=2}^{n-1}\left(P_{a, 0}\left(a_{i}\right)+b\right) .
$$

We then choose $a_{1}, \ldots, a_{n-1}$ so that for $b$ of a certain form, the apparent probability that $P_{a, b}$ has the desired properties is positive. Since $\Delta_{a, b}$ factors as a product of linear polynomials in $b$, it is tractable to carry out the sieving argument to confirm that the apparent probability is correct; however, in lieu of doing the sieving by hand, we appeal to a very general squarefree sieve set up by Helfgott [10. (The argument is a bit simpler if one does not insist on the number of real roots; see Proposition 2.1.) 
From Theorem 1.1] we obtain at once the following corollary.

Corollary 1.3. With notation as in Theorem 1.1, there exist infinitely many number fields $K$ of degree $n=r+2 s$ and signature $(r, s)$ such that the Galois closure $L$ of $K$ has Galois group $S_{n}$ over $\mathbb{Q}$ and the discriminant of $K$ is squarefree and not divisible by any of the primes in $S$. More precisely, for some $c>0$ (depending on $n$ and $S$ ), the number of distinct such discriminants produced in the range $[-N, N]$ is at least $c N^{1 /(n-1)}$.

As noted earlier, these conditions ensure that $L$ is an unramified $A_{n}$-extension of $\mathbb{Q}(\sqrt{\Delta(K)})$. We thus obtain the following.

Corollary 1.4. Let $S$ be a finite set of primes. For each $n \geq 3$, there exist infinitely many real quadratic fields unramified above the primes in $S$ and admitting an $A_{n}$ extension unramified at all finite and infinite primes. More precisely, for some $c>0$ (depending on $n$ and $S$ ), the number of distinct such discriminants produced in the range $[-N, N]$ is at least $c N^{1 /(n-1)}$.

Corollary 1.4 generalizes work of several authors. Uchida [16] and Yamamoto 17. proved independently that there exist infinitely many real and infinitely many imaginary quadratic fields with $A_{n}$-extensions unramified at all finite primes. They did so by constructing number fields of the form $\mathbb{Q}[x] /\left(x^{n}+a x+b\right)$ and showing that under suitable conditions on $a$ and $b$, their normal closures are frequently $S_{n^{-}}$ extensions of $\mathbb{Q}$ unramified over a quadratic subfield. However, these number fields fail to be totally real for $n \geq 4$, so the construction does not produce the result of Corollary 1.4. The case $n=5$ of Corollary 1.4 was obtained by Yamamura 18; however, Yamamura's construction produces number fields of the form $\mathbb{Q}[x] /(P(x))$ where the polynomial $P$ is of a very special form which does not generalize to higher degree. (Note that none of the aforementioned constructions guarantee squarefree discriminants.)

One may also deduce from Corollary 1.3 that the number of $S_{n}$-number fields of signature $(r, s)$ with discriminant in $[-N, N]$ is bounded below by $c N^{1 /(n-1)}$. This is far inferior to what can be shown using other methods: for $n \leq 5$ the number of such fields is known to be $O(N)$ by work of Bhargava 2, 3, while for larger $n$, Ellenberg and Venkatesh [5] have given a lower bound of $c N^{1 / 2+1 / n^{2}}$. However, the fields we produce have squarefree discriminants and monogenic rings of integers, which is not guaranteed by these other constructions (although Bhargava's method should allow for squarefree sieving; see [4]).

\section{Construction of Squarefree Discriminants}

In this section, we give the proof of a weaker form of Theorem 1.1, in which we do not control the number of real roots. This helps isolate the essential features of the construction.

Proposition 2.1. Let $n \geq 2$ be an integer and let $S$ be a finite set of primes. Then there exist infinitely many monic irreducible polynomials $P(x)$ of degree $n$ with integer coefficients such that $\Delta(P)$ is squarefree and not divisible by any of the primes in $S$. More precisely, for some $c>0$ (depending on $n$ and $S$ ), the number of distinct discriminants produced in the range $[-N, N]$ is at least $c N^{1 /(n-1)}$.

The approach to the proof is to consider polynomials $P_{a, b}$ as in the introduction with $a_{1}, \ldots, a_{n-1} \in \mathbb{Z}$ chosen to satisfy the following conditions. 
(i) The polynomial $P_{a, 0}$ has integral coefficients.

(ii) For each $p \in S$, there exists $b_{p} \in \mathbb{Z}$ such that $\Delta_{a, b_{p}}$ is not divisible by $p$.

(iii) For each $p \notin S$, there exists $b \in \mathbb{Z}$ such that $\Delta_{a, b}$ is not divisible by $p^{2}$. (This ensures that $\Delta_{a, b}$ is squarefree with positive probability.)

(iv) There exist $p_{1} \notin S$ and $b_{1} \in \mathbb{Z}$ such that $P_{a, b_{1}}$ is irreducible modulo $p_{1}$; in particular, $\Delta_{a, b_{1}}$ is not divisible by $p_{1}$. (This ensures that $P_{a, b_{1}}$ is irreducible for $b \equiv b_{1}\left(\bmod p_{1}\right)$.)

(v) The polynomials $n^{n} P_{a, 0}\left(a_{1} / n\right)+n^{n} b, P_{a, 0}\left(a_{2}\right)+b, \ldots, P_{a, 0}\left(a_{n-1}\right)+b$ in $b$ are pairwise coprime. (This ensures that $\Delta_{a, b}$ is squarefree as a polynomial in $b$.

Given such a choice of $a_{1}, \ldots, a_{n-1}$, let $T$ be the set of all $b \in \mathbb{Z}$ such that $b \equiv b_{p}$ $(\bmod p)$ for each $p \in S$ and $b \equiv b_{1}\left(\bmod p_{1}\right)$. For $b \in T, P_{a, b}$ is irreducible with integer coefficients, $\Delta_{a, b}$ is not divisible by any prime in $S$, and by a sieving argument (see Lemma 2.2 below), $\Delta_{a, b}$ is squarefree with positive probability. Since no value of $\Delta_{a, b}$ occurs for more than $n-1$ choices of $b$, by taking $b$ of size $O\left(N^{1 /(n-1)}\right)$, we obtain at least $c N^{1 /(n-1)}$ squarefree values of $\Delta_{a, b}$ in the range $[-N, N]$ for some fixed $c>0$.

The sieving argument required in this argument is essentially no harder than proving the density of squarefree integers; however, we have nothing to add to Helfgott's general presentation of the squarefree sieve, so we defer to it instead.

Lemma 2.2. Let $m$ be a positive integer. Suppose that $c_{1}, d_{1}, \ldots, c_{k}, d_{k} \in \mathbb{Z}$ are such that the polynomial $A(x)=\left(c_{1} x+d_{1}\right) \cdots\left(c_{k} x+d_{k}\right)$ is squarefree. For $p$ prime, put

$$
a(p)=\frac{\#\left\{x \in\left\{0, \ldots, p^{2}-1\right\}: A(x) \not \equiv 0\left(\bmod p^{2}\right)\right\}}{p^{2}} .
$$

Suppose that $a(p)>0$ for all $p$. Then

$$
\lim _{N \rightarrow \infty} \frac{\#\{x \in\{-N, \ldots, N\}: A(x) \text { is squarefree }\}}{2 N+1}=\prod_{p} a(p)>0 .
$$

Note that $\prod_{p} a(p)$ converges to a positive limit because $1-k / p^{2} \leq a(p) \leq 1$ for all $p$ not dividing $\prod_{i<j}\left(c_{i} d_{j}-c_{j} d_{i}\right)$.

Proof. See [10, Proposition 3.4].

It remains to check that conditions (i)-(v) can be enforced via certain congruence conditions on $a_{1}, \ldots, a_{n-1}$. To make these congruences compatible with the requirement that the derivative of $P_{a, b}$ have only rational roots, we need some auxiliary calculations.

Lemma 2.3. Let $p_{0}$ be a prime not dividing $n(n-1)$. Then there exist infinitely many primes $p_{1}$ modulo which the polynomial $R(x)=x^{n}-p_{0}^{n-1} x+p_{0}$ is irreducible and its derivative $R^{\prime}(x)=n x^{n-1}-p_{0}^{n-1}$ splits into distinct linear factors.

Proof. The polynomial $R^{\prime}$ has splitting field $L=\mathbb{Q}\left(\zeta_{n-1}, n^{1 /(n-1)}\right)$, in which $p_{0}$ does not ramify because $p_{0}$ does not divide $n(n-1)$. Thus $R$ is an Eisenstein polynomial with respect to any prime above $p_{0}$ in $L$; in particular, $R$ is irreducible over $L$. By the Chebotarev density theorem, there exist infinitely many prime ideals of $L$ of absolute degree 1 modulo which $R$ is irreducible; the norm of any such prime ideal is a prime number of the desired form. 
Lemma 2.4. For any field $F$ of characteristic zero, there exist $a_{1}, \ldots, a_{n-1} \in F$ such that the values $P_{a, 0}\left(a_{1} / n\right), P_{a, 0}\left(a_{2}\right), \ldots, P_{a, 0}\left(a_{n-1}\right)$ are pairwise distinct.

Proof. For $a_{1}, \ldots, a_{n-1} \in \mathbb{Q}$ with $a_{1}<\cdots<a_{n-1}$,

$$
P_{a, 0}\left(a_{n-1}\right)-P_{a, 0}\left(a_{n-2}\right)=\int_{a n-1}^{a_{n-2}}\left(t-a_{1} / n\right)\left(t-a_{2}\right) \cdots\left(t-a_{n-1}\right) d t \neq 0
$$

because the integrand has constant sign over the interval. Consequently, the polynomial $P_{a, 0}\left(a_{n-1}\right)-P_{a, 0}\left(a_{n-2}\right)$ in $a_{1}, \ldots, a_{n-1}$ is nonzero. By similar reasoning, each of the factors of the polynomial

$$
\prod_{i=2}^{n-1}\left(P_{a, 0}\left(a_{i}\right)-P_{a, 0}\left(a_{1} / n\right)\right) \prod_{2 \leq i<j \leq n-1}\left(P_{a, 0}\left(a_{j}\right)-P_{a, 0}\left(a_{i}\right)\right)
$$

in $a_{1}, \ldots, a_{n-1}$ is not zero, so the product is not the zero polynomial either. Since $F$ is infinite, the claim follows.

We are now ready to complete the proof of Proposition 2.1.

Proof of Proposition 2.1. By the previous discussion, it is sufficient to exhibit $a_{1}, \ldots, a_{n-1} \in \mathbb{Z}$ for which conditions (i)-(v) hold. Choose $p_{0}, R, p_{1}$ as in Lemma [2.3 making sure that $p_{1} \notin S \cup\{1, \ldots, n\}$. Apply Lemma 2.4 to choose $a_{1}^{\prime}, \ldots, a_{n-1}^{\prime} \in \mathbb{Q}$ for which

$$
P_{a^{\prime}, 0}\left(a_{1}^{\prime} / n\right), P_{a^{\prime}, 0}\left(a_{2}^{\prime}\right), \ldots, P_{a^{\prime}, 0}\left(a_{n-1}^{\prime}\right)
$$

are pairwise distinct. We can then choose a prime $p_{2} \notin S \cup\left\{1, \ldots, n, p_{1}\right\}$ for which the values in (2.5) have well-defined and distinct reductions modulo $p_{2}$. We now choose $a_{1}, \ldots, a_{n-1}$ according to the following conditions (which is evidently possible).

(a) The integer $a_{1}$ is coprime to $n$ and is divisible by $n-1$ and by all primes of $S$ not dividing $n$. The integers $a_{2}, \ldots, a_{n-1}$ are divisible by $n !$ and by all primes in $S$.

(b) The quantities $a_{1} / n, a_{2}, \ldots, a_{n-1}$ are congruent modulo $p_{1}$ to the roots of $R^{\prime}(x)$ (in some order).

(c) We have $a_{i} \equiv a_{i}^{\prime}\left(\bmod p_{2}\right)$ for $i=1, \ldots, n-1$.

We then have

$$
Q_{a}(x) \equiv\left(n x-a_{1}\right) x^{n-2}=n x^{n-1}-a_{1} x^{n-2}(\bmod n !),
$$

so the coefficient of $x^{m-1}$ in $Q_{a}(x)$ is divisible by $m$ for $m=1, \ldots, n$. Consequently, $P_{a, 0}$ has integer coefficients. This verifies (i). Suppose next that $p$ is a prime which either belongs to $S$ or is at most $n$. For $b \in \mathbb{Z}, P_{a, 0}\left(a_{i}\right)+b \equiv b(\bmod p)$ for $i=2, \ldots, n-1$, while

$$
n^{n} P_{a, 0}\left(a_{1} / n\right)+n^{n} b \equiv \begin{cases}a_{1}^{n}(\bmod p) & \text { if } p \text { divides } n \\ n^{n} b(\bmod p) & \text { otherwise. }\end{cases}
$$

Consequently, $\Delta_{a, 1}$ is not divisible by $p$; this verifies (ii), as well as (iii) for primes $p \leq n$. For $p>n$ not in $S$, there are at least $p-n+1$ choices of $b \in\{0, \ldots, p-1\}$ for which $\Delta_{a, b}$ is not even divisible by $p$, since each linear factor of $\Delta_{a, b}$ rules out exactly one choice of $b$. This verifies (iii). Since (b) implies (iv) and (c) implies (v), the needed conditions are enforced, so the proof described above goes through. 


\section{Controlling ReAl roots}

Note that the proof of Proposition 2.1 cannot be used to deduce Theorem [1.1 because it does not allow any control of the number of real roots of the polynomial $P_{a, b}$. Indeed, the function $P_{a, 0}(x)$ is monotonic for $x$ large and for $x$ small, so $P_{a, b}$ has at most 2 real roots for $|b|$ sufficiently large. To obtain Theorem 1.1, we must modify the proof of Proposition 2.1 so that $b$ can be chosen to be a rational number within a suitable interval.

Proof of Theorem 1.1. Define $p_{0}, R, p_{1}, p_{2}, a_{1}^{\prime}, \ldots, a_{n-1}^{\prime}$ as in the proof of Proposition 2.1. Let $U$ be the set of $A=\left(A_{1}, \ldots, A_{n-1}\right) \in \mathbb{R}^{n-1}$ for which there exists $B \in \mathbb{R}$ such that the polynomial $P_{A, B}$ has exactly $r$ real roots; the set $U$ is easily seen to be open and nonempty. It is also homogeneous: if $\left(A_{1}, \ldots, A_{n-1}\right)$ belongs to $U$, then so does $\left(\lambda A_{1}, \ldots, \lambda A_{n-1}\right)$ for any $\lambda \in \mathbb{R}$. In other words, $U$ is the inverse image in $\mathbb{R}^{n-1}$ of a nonempty open subset of the $(n-2)$-dimensional projective space over $\mathbb{R}$. In that space, the images of the tuples consisting of integers satisfying conditions (a)-(c) of the proof of Proposition 2.1 form a dense subset; we may thus choose $\left(A_{1}, \ldots, A_{n-1}\right) \in U$ satisfying these conditions. Fix such a choice hereafter; there then exists a nonempty interval $I$ such that $P_{A, B}$ has exactly $r$ real roots for all $B \in I$.

We wish to take $a_{i}=A q$ for some positive integer $q \equiv 1\left(\bmod n ! p_{1} p_{2} \prod_{p \in S} p\right)$. These still satisfy conditions (a)-(c) of the proof of Proposition 2.1, so conditions (i) $-(\mathrm{v})$ are enforced. We may then sieve again (see Lemma 3.1) to show that among $b$ of this form congruent to $b_{p}$ modulo $p$ for each $p \in S$ and congruent to $b_{1}$ modulo $p_{1}$, those for which $\Delta_{a, b}$ is squarefree occur with positive probability. Since the map $b \rightarrow \Delta_{a, b}$ is at most $(n-1)$-to-one, if we consider all $q \leq N^{1 /(n-1)}$, the number of squarefree discriminants obtained is at least $1 /(n-1)$ times the maximum number of squarefree values of $\Delta_{a, b}$ achieved for any single choice of $q$ in this range. This yields a lower bound of $c N^{1 /(n-1)}$ discriminants of absolute value at most $N$, proving Theorem 1.1 .

In this case, the sieving argument needed is an easy (because we only consider polynomials composed of linear factors) variant of the squarefree sieving for homogeneous binary forms introduced by Greaves [8]. However, we again prefer to apply Helfgott's machine rather than get into the details.

Lemma 3.1. Let $k, n, t$ be positive integers, and let $I$ be a nonempty open interval. Suppose that $c_{1}, d_{1}, \ldots, c_{k}, d_{k} \in \mathbb{Z}$ are such that the polynomial $A(x, y)=\left(c_{1} x+\right.$ $\left.d_{1} y\right) \cdots\left(c_{k} x+d_{k} y\right)$ is squarefree. For $p$ prime, if $p$ divides $t$, then put

$$
a(p)=\frac{\#\left\{x \in\left\{0, \ldots, p^{2}-1\right\}: A(x, 1) \not \equiv 0\left(\bmod p^{2}\right)\right\}}{p^{2}} ;
$$

otherwise, put

$$
a(p)=\frac{\#\left\{(x, y) \in\left\{0, \ldots, p^{2}-1\right\}^{2}: \operatorname{gcd}(x, y)=1, A\left(x, y^{n}\right) \not \equiv 0\left(\bmod p^{2}\right)\right\}}{p^{2}(p-1)^{2}} .
$$

Suppose that $a(p)>0$ for all $p$. Define

$$
S_{N}=\left\{(x, y) \in \mathbb{Z}^{2}: 1 \leq y \leq N, y \equiv 1(\bmod t), \operatorname{gcd}(x, y)=1, x / y^{n} \in I\right\} .
$$


Then

$$
\lim _{N \rightarrow \infty} \frac{\#\left\{(x, y) \in S_{N}: A\left(x, y^{n}\right) \text { is squarefree }\right\}}{\# S_{N}}=\prod_{p} a_{p}>0 .
$$

Proof. This follows from [10. Corollary 3.3] modulo matching up notation, which we now explain (following the model of [10, Proposition 3.5]). We define a soil in the sense of [10, §3.2] by taking

$$
\begin{gathered}
\mathscr{P}=\{\text { primes in } \mathbb{Z}\}, \quad \mathscr{A}=S_{N}, \\
r(x, y)=\left\{p \in \mathscr{P}: p^{2} \mid A\left(x, y^{n}\right)\right\}, \quad f(x, y, d)= \begin{cases}1 & d=\emptyset \\
0 & \text { otherwise }\end{cases}
\end{gathered}
$$

Put $X=\# S_{N}$; note that $X$ is asymptotic to a constant times $N^{n+1}$ as $N \rightarrow \infty$. For $d \subset \mathscr{P}$ finite, write $h(d)$ for $\prod_{p \in d} p^{2}$; this function evidently satisfies conditions (h1) and (h2) of [10, §3.2]. Let $K(d)$ be the number of $(x, y) \in \mathscr{A}$ with $d \subseteq r(x, y)$; since there are only finitely many primes that can divide more than one factor of $A\left(x, y^{n}\right)$, we have $K(d) \leq C_{0} X / h(d)$ for some $C_{0}>0$ (dependent on everything but $N$ and $d$ ). This means that condition (A1) of [10, §3.2] holds with $C_{1}=1, C_{2}=0$.

For $d$ with $h(d) \leq N^{1 / 4}$, put $g(d, \emptyset)=h(d) \prod_{p \in d} a(p)$; we may then write

$$
K(d)=X \frac{g(d, \emptyset)}{h(d)}+r_{d, \emptyset}
$$

with $\left|r_{d, \emptyset}\right| \leq N h(d) \leq N^{5 / 4}$. (Namely, for each $y$, we are counting values of $x$ in an interval satisfying certain congruence conditions modulo $h(d)$; the difference between this count and the expected value is at most 1 in each congruence class.) By [10, Corollary 3.3], the difference

$$
\prod_{p} a_{p}-\frac{\#\left\{(x, y) \in S_{N}: A\left(x, y^{n}\right) \text { is squarefree }\right\}}{X}
$$

is bounded in absolute value by

$$
C_{1} \sum_{d: h(d)>N^{1 / 4}} \frac{3^{\# d}}{h(d)}+X^{-1} \sum_{d: h(d) \leq N^{1 / 4}}\left|r_{d, \emptyset}\right|+X^{-1} C_{2} \sum_{p>N} \frac{X}{p^{2}}
$$

for some $C_{1}, C_{2}>0$ independent of $N$. The first and third terms evidently tend to 0 as $N \rightarrow \infty$; the second does also because it is bounded above by $X^{-1} N^{1 / 4} N^{5 / 4} \leq$ $C_{3} N^{-n+1 / 2}$ for some $C_{3}>0$ independent of $N$. This proves the claim.

\section{FURTHER REMARKS}

We have not attempted to improve upon the lower bound of $c N^{1 /(n-1)}$ in Theorem 1.1] or its corollaries. Nakagawa [13], 14] attempted to show that the number of real quadratic fields admitting $A_{n}$-extensions unramified at all finite primes with discriminants in $[-N, N]$ is at least $c_{n} N^{(n+1) /(2 n-2)}$ for all $n$, but these proofs were later retracted. This order of growth would be obtained if one could show that the discriminant of an arbitrary (not necessarily monic) integer polynomial of degree $n$ is squarefree with positive probability and that the number of distinct discriminants obtained is at least a fixed positive fraction of the number of polynomials considered. Even if one assumes the $a b c$ conjecture, so that the first issue is resolved by Poonen's theorem, the second issue remains: for any construction of polynomials 
involving more than one free parameter, it is nontrivial to ensure that the same discriminant does not occur too many times.

It would also be of interest to extend our results by allowing restrictions on the splitting of some finite places. Our method is unsuitable for this purpose: a splitting requirement would constitute an additional restriction on the reduction of a polynomial modulo specific primes, which is hard to integrate with the requirement that the derivative of the polynomial have rational roots modulo any prime. Indeed, for primes less than $n$, certain splitting requirements on the polynomial alone (e.g., that it factors completely) are incompatible with having a squarefree discriminant, because there are not enough residual roots available for them to be pairwise distinct.

\section{ACKNOWLEDGMENTS}

This paper is a revised version of our unpublished 2003 preprint "Number fields with squarefree discriminant and prescribed signature", written while the author was supported by an NSF Postdoctoral Fellowship. The revisions were made during the spring 2010 MSRI program in arithmetic statistics. The author thanks Manjul Bhargava, Chandan Singh Dalawat, Noam Elkies, and Harald Helfgott for helpful discussions and especially Manju Bhargava for encouraging the author to revisit the 2003 manuscript.

\section{REFERENCES}

[1] A. Ash, J. Brakenhoff, and T. Zarrabi, Equality of polynomial and field discriminants, Exper. Math. 16 (2007), 367-374. MR2367325(2008i:11129)

[2] M. Bhargava, The density of discriminants of quartic rings and fields, Ann. of Math. (2) 162 (2005), 1031-1063. MR2183288 (2006m:11163)

[3] M. Bhargava, The density of discriminants of quintic rings and fields, Ann. of Math. (2) 172 (2010), 1559-1591. MR2745272

[4] M. Bhargava, The geometric squarefree sieve and unramified nonabelian extensions of quadratic fields, preprint (2011).

[5] J.S. Ellenberg and A. Venkatesh, The number of extensions of a number field with fixed degree and bounded discriminant, Ann. of Math. (2) 163 (2006), 723-741. MR2199231 (2006j:11159)

[6] J. Elstrodt, F. Grunewald, and J. Mennicke, On unramified $A_{m}$-extensions of quadratic number fields, Glasgow Math. J. 27 (1985), 31-37. MR819826 (87e:11122)

[7] A. Granville, $A B C$ allows us to count squarefrees, Intl. Math. Res. Notices 1998, 991-1009. MR.1654759 (99j:11104)

[8] G. Greaves, Power-free values of binary forms, Quart. J. Math. Oxford 43 (1992), 45-65. MR1150469 (92m:11098)

[9] G.H. Hardy and E.M. Wright, An Introduction to the Theory of Numbers, fourth edition, Oxford University Press (London), 1965.

[10] H.A. Helfgott, On the square-free sieve, Acta Arith. 115 (2004), 349-402. MR 2099831 (2005h:11211)

[11] C. Hooley, On the power free values of polynomials, Mathematika 14 (1967), 21-26. MR0214556 (35:5405)

[12] T. Kondo, Algebraic number fields with the discriminant equal to that of a quadratic number field, J. Math. Soc. Japan 47 (1995), 31-36. MR1304187(95h:11121)

[13] J. Nakagawa, Binary forms and orders of algebraic number fields, Invent. Math. 97 (1989), 219-235; erratum, Invent. Math. 105 (1991), 443. MR1001839 (90k:11042)

[14] J. Nakagawa, Binary forms and unramified $A_{n}$-extensions of quadratic fields, J. Reine Angew. Math. 406 (1990), 167-178; correction, J. Reine Angew. Math. 413 (1991), 220. MR1048239 (91d:11037) MR:1089804 (91j:11019) 
[15] B. Poonen, Squarefree values of multivariate polynomials, Duke Math. J. 118 (2003), 353373. MR,1980998 (2004d:11094)

[16] K. Uchida, Unramified extensions of quadratic number fields, II, Tôhoku Math. J. 22 (1970), 220-224. MR0272760 (42:7641)

[17] Y. Yamamoto, On unramified Galois extensions of quadratic number fields, Osaka J. Math. 7 (1970), 57-76. MR0266898(42:1800)

[18] K. Yamamura, On unramified Galois extensions of real quadratic number fields, Osaka J. Math. 23 (1986), 471-478. MR856901 (88a:11112)

Department of Mathematics, Massachusetts Institute of Technology, 77 MassachuSetts Avenue, Cambridge, Massachusetts 02139 - and - Department of Mathematics, University of California, San Diego, 9500 Gilman Drive \#0112, La Jolla, California 92093

E-mail address: kedlaya@mit.edu

E-mail address: kedlaya@ucsd.edu 